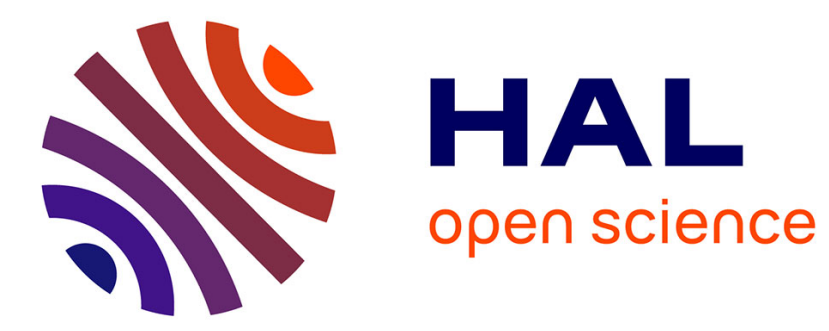

\title{
Chromosome Y et détermination du sexe
}

François Rouyer, Jean Weissenbach

\section{To cite this version:}

François Rouyer, Jean Weissenbach. Chromosome Y et détermination du sexe. Reproduction Nutrition Development, 1990, 30 (Suppl1), pp.27s-38s. hal-00899306

\section{HAL Id: hal-00899306 https://hal.science/hal-00899306}

Submitted on 1 Jan 1990

HAL is a multi-disciplinary open access archive for the deposit and dissemination of scientific research documents, whether they are published or not. The documents may come from teaching and research institutions in France or abroad, or from public or private research centers.
L'archive ouverte pluridisciplinaire HAL, est destinée au dépôt et à la diffusion de documents scientifiques de niveau recherche, publiés ou non, émanant des établissements d'enseignement et de recherche français ou étrangers, des laboratoires publics ou privés. 


\title{
Chromosome $Y$ et détermination du sexe
}

\author{
J Weissenbach, F Rouyer \\ Unité de Recombinaison et Expression Génétique, INSERM U163, CNRS UA271, \\ Institut Pasteur, 25, rue du Docteur-Roux, 75015 Paris, France
}

(28 Réunion de la Société Française pour l'Étude de la Fertilité; Paris, 19-21 octobre 1989)

Résumé - La structure puis la fonction du chromosome $Y$ humain ont été abordées depuis quelques années par les techniques de génétique moléculaire. L'obtention de nombreuses sondes ADN du chromosome $Y$ et l'analyse, à l'aide de ces sondes, de ce chromosome chez des patients présentant des anomalies de la différenciation sexuelle ont permis de construire une carte par délétion localisant le locus TDF (testis determining factor) dans la partie distale du bras court. Ces études ont abouti récemment à l'obtention d'un gène candidat pour le locus TDF. Cependant l'existence d'un gène apparenté sur le chromosome $X$ remet en cause l'hypothèse simple du gène de différenciation testiculaire primaire porté par le chromosome $Y$.

La mise en évidence d'une région d'homologie stricte entre les chromosomes $X$ et $Y$ à l'extrémité des bras courts a permis de caractériser une autre grande propriété des chromosomes sexuels. Les séquences présentes dans cette région n'ont qu'un caractère partiel de liaison au sexe et peuvent être échangées entre $X$ et $Y$ lors de la méiose mâle, d'où leur dénomination de pseudoautosomique. L'analyse génétique de la région pseudoautosomique et la détermination de sa taille physique montrent qu'elle subit en méiose mâle, un taux de recombinaison méiotique environ 20 fois plus élevé que le reste du génome.

En 1966, Ferguson-Smith avait proposé qu'une crossing-over anormal était à l'origine des deux grands types d'inversions de sexe observés : hommes $X X$ et femmes $X Y$. L'étude de la ségrégation des séquences pseudoautosomiques chez ces patients a montré la validité de cette hypothèse de même que l'existence d'inversions de sexe qui semblent indépendantes du locus TDF.

La construction d'une carte détaillée du chromosome $Y$ humain rend possible la recherche de nouveaux gènes (antigène $H-Y, g e ̀ n e(s)$ de fertilité). Enfin, la mise en évidence de nombreuses régions d'homologie entre les chromosomes sexuels témoigne de leur origine commune et permettra peut-être de mieux comprendre la mise en place des mécanismes de différenciation sexuelle et d'inactivation du chromosome $X$ au cours de l'évolution des mammifères.

chromosome $Y$ / différenciation testiculaire / séquences pseudoautosomiques / hommes XX / femmes $X Y$

Summary $-Y$ chromosome and sex determination. The structure and function of the human $Y$ chromosome have been approached by molecular genetics. The use of Y-derived DNA probes to analyze the $Y$ chromosome of patients with sexual differentiation abnormalities allowed the construction of a deletion map localizing TDF (testis determining factor) in the terminal part of the short arm. These studies culminated recently in the isolation of a candidate gene for the TDF locus. Nevertheless, the existence of a related locus on the $X$ chromosome does not fit with the simple hypothesis of a single $Y$-located gene for testis differentiation.

The finding of a strictly homologous region at the tip of the short arms of $X$ and $Y$ chromosomes allowed the characterization of another major property of the sex chromosomes. These so called pseudoautosomal DNA sequences show only a partial sex linkage and can be exchanged between $X$ and $Y$ chromosomes during male meiosis. Genetic analysis and determination of the physical length of the pseudoautosomal region show that it is subjected in male meiosis, to a recombination rate about 20 times higher than the mean value of the human genome.

In 1966, Ferguson-Smith proposed that an abnormal cross-over was responsible for both types of sex inversions in humans: $X X$ males and $X Y$ females. Segregation analysis of the pseudoautosomal 
loci in these patients has demonstrated the validity of this hypothesis and shown the existence of sex inversions unrelated to the TDF locus.

The construction of a detailed map of the human $Y$ chromosome enables the search for new genes ( $H-Y$ antigen, fertility gene(s)). The finding of several homologous regions on the sex chromosomes confirms the hypothesis of a common origin and should help to understand the establishment of sex determination and $X$ inactivation mechanisms during mammalian evolution.

Y chromosome / testis differentiation / pseudoautosomal sequences / XX males / XY females

L'utilisation des techniques de l'ADN recombinant a fait exploser le volume des connaissances dans le domaine de la génétique humaine. Des gènes comme ceux de la prédisposition au rétinoblastome ou de la myopathie de Duchenne, qui n'avaient, il y a encore quelques années, qu'une définition formelle, ont pu être isolés physiquement grâce à la méthodologie de la génétique dite inverse (Friend et al, 1986; Monaco et al, 1986; Orkin, 1986). De tels travaux sont aussi à l'origine de nouveaux concepts en biologie et de nouvelles avancées sur le plan diagnostic.

La technologie de l'ADN recombinant a également permis d'aborder des questions où l'analyse génétique n'avait pas de prise. La détermination du sexe chez les mammifères est un exemple qui illustre de manière particulière la puissance des méthodes et des outils moléculaires développés depuis une quinzaine d'années. Le présent article reprend les principales phases de l'analyse moléculaire du processus de détermination du sexe, qui a abouti, il y a deux ans, à l'isolement d'un gène qui pourrait avoir pour fonction d'initier le processus de differenciation testiculaire (Page et al, 1987c).

\section{CARTOGRAPHIE DU FACTEUR DE DÉTERMINATION TESTICULAIRE}

On sait depuis la fin des années cinquante qu'un locus dominant du chromosome $Y$ est responsable de la détermination du sexe masculin (Ford et al, 1959; Jacobs et
Strong, 1959). Au cours des vingt années qui ont suivi, deux catégories majeures d'exceptions à ce dogme ont été observées. II s'agit d'une part de sujets de sexe masculin avec deux chromosomes $X$ (hommes XX) (de la Chapelle, 1981) et d'autre part de sujets de sexe féminin avec un caryotype $46, X Y$ (femmes $X Y$ ) (Simpson et al, 1971; Rosenfeld et al, 1979). Alors que chez les hommes $X X$ les gonades sont des testicules azoospermiques, les gonades des femmes $X Y$ sont réduites à des bandelettes indifférenciées (dysgénésies gonadiques pures) et le terme de femmes $X Y$ correspond plutôt à un abus de langage. La plupart de ces inversions de sexe sont sporadiques; leur incidence est de $1 / 20000$ pour les hommes $X X$ et de $1 / 500000$ pour les femmes $X Y$. De nombreuses hypothèses ont été avancées pour expliquer ces phénotypes. La plus séduisante, proposée par FergusonSmith, consiste à supposer que les deux anomalies résultent d'un échange méiotique anormal entre les chromosomes sexuels paternels (Ferguson-Smith, 1966). Dans cette hypothèse, la partie du chromosome $\mathrm{Y}$ incluant le locus responsable de la détermination du sexe (TDF = testisdetermining factor) serait mobilisée dans un crossing-over anormal entre les chromosomes $X$ et $Y$. Le chromosome $Y$ remanié aurait perdu le locus TDF et deviendrait associé à un phénotype femelle, alors que le chromosome $X$ remanié aurait acquis ce locus et serait associé à un phénotype mâle. 
Les premiers indices expérimentaux de ce modèle ont été obtenus près de 20 ans plus tard. D'abord, une analyse cytogénétique en prométaphase des chromosomes de mâles $X X$ suggérait que la partie terminale du bras court du chromosome $Y$ se retrouvait à l'extrémité du bras court du chromosome X (Evans et al, 1979; Magenis et al, 1982). Peu après, l'utilisation de sondes d'ADN spécifiques du chromosome $Y$ nous a permis de montrer la présence d'un fragment de chromosome $Y$ chez la plupart des mâles $X X$ analysés (Guellaën et al, 1984). De plus ce fragment chromosomique était de taille variable à une des extrémités. Ceci suggérait que TDF devait plutôt être localisé dans une partie invariable de ce segment chromosomique commune à tous mâles $X X$ relevant de cette étiologie et ouvrait donc la voie à une cartographie par délétion du facteur masculinisant (Vergnaud et al, 1986; Müller et al, 1986; Affara et al, 1987). Par hybridation in situ, il fut ensuite montré que ce fragment était localisé à l'extrémité du chromosome $X$ chez les mâles $X X$ (Andersson et al, 1986; Buckle et al, 1987; Magenis et al, 1987). Des analyses moléculaires de cas de féminité $X Y$ avec délétions $Y p-$ montraient par ailleurs que les loci $Y$-spécifiques délétés chez ces patientes étaient identiques à ceux présents chez les mâles $X X$ (Disteche et al, 1986; Müller et al, 1986; Affara et al, 1987). Ces résultats concordaient et s'interprétaient par une localisation du gène TDF sur la partie distale du bras court du chromosome Y. L'établissement de ces différentes cartes par délétion postulait néanmoins un arrangement unique des loci le long du chromosome Y. Du fait de l'absence de crossing-over pour la majeure partie de ce chromosome, il ne pouvait être exclu que de petits réarrangements compatibles avec le maintien de l'expression de ses fonctions aient créé plusieurs ordres de loci. A côté d'un ordre majoritaire, quel- ques variants ont en effet été observés (Affara et al, 1986a; Disteche et al, 1986; Brøndum Nielsen et al, 1988; Scherer et al, 1989). Ces différents ordres peuvent apparemment dériver les uns des autres par une simple inversion du bras court. Mais ces inversions n'ont pas modifié la localisation de TDF dans la partie distale du bras court du chromosome $Y$. Ces premières cartes par délétion n'ont cependant pas réussi à définir une borne distale au locus TDF.

\section{RÉGION PSEUDOAUTOSOMIQUE ET CROSSING-OVER ENTRE LES CHROMOSOMES $X$ ET $Y$}

L'isolement d'un nombre de plus en plus important de sondes du chromosome $Y$ nous a permis d'identifier des séquences d'ADN présentant un dégré d'homologie absolu entre les chromosomes $X$ et $Y$ humains et localisées dans la partie terminale des bras courts (Simmler et al, 1985). Certaines de ces sondes détectent des fragments polymorphes dont la ségrégation a pu être suivie par une analyse familiale. Cette étude montre que chacun des allèles paternels d'un même locus polymorphe ségrège aussi bien dans la descendance mâle que femelle (Simmler et al, 1985). Une recombinaison entre un tel locus et TDF doit donc avoir eu lieu à la méiose. Un travail similaire a été réalisé, simultanément par un groupe écossais (Cooke et al, 1985) et, peu après, l'équipe de Goodfellow montrait que le gène MIC2, codant pour l'antigène de surface 12E7, n'avait qu'une liaison partielle du sexe (Goodfellow et al, 1986). L'ensemble de ces résultats ont permis de définir une nouvelle région constituée de loci qui ne sont plus strictement liés au sexe bien que localisés sur les chromosomes sexuels. En raison de ce caractère apparemment auto- 
somique, ces loci ont reçu le qualificatif de "pseudoautosomique" (Burgoyne, 1982). II est à présent convenu d'appeler région pseudoautosomique cette partie strictement homologue de la région terminale des bras courts des chromosomes sexuels humains. TDF étant spécifique du chromosome $Y$, une localisation pseudoautosomique paraît exclue et la région pseudoautosomique constitue donc la borne distale du locus de différenciation testiculaire (fig 1).

\section{CARTOGRAPHIE FINE DU LOCUS TDF ET ISOLEMENT D'UN GĖNE CANDIDAT}

Une étude systématique d'un grand nombre d'inversions de sexe, à l'aide de

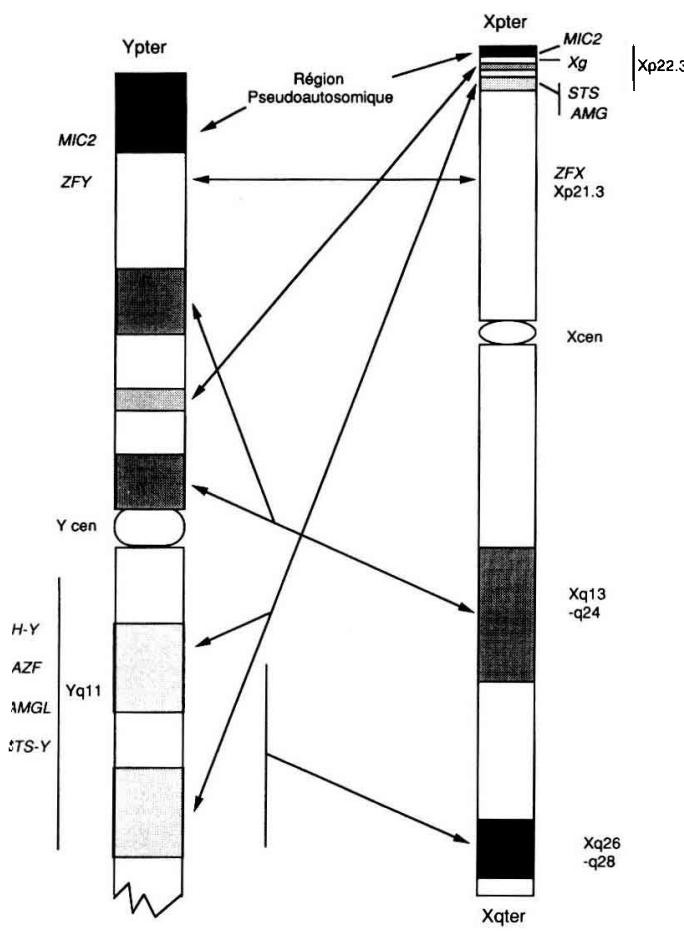

Fig 1. Schéma des homologies de séquences d'ADN entre les chromosomes $X$ et $Y$ humains. plus d'une centaine de sondes spécifiques du chromosome $\mathrm{Y}$, a permis au groupe de Page de définir un intervalle limité à 140 $\mathrm{kb}$ et contenant au moins une partie du locus TDF (Page et al, 1987c). Quelques sondes de cet intervalle détectent des séquences conservées chez tous les mammifères euthériens examinés. Des ADNc de l'homologue murin de ce gène ont été isolés et séquencés (Mardon et Page, 1989). La séquence protéique ainsi déduite apparaît constituée de deux domaines séparés par une région charnière. La partie $\mathrm{N}$ terminale est très riche en résidus acides, alors que la partie $\mathrm{C}$ terminale est constituée de motifs caractéristiques à cystéines et histidines connus sous le nom de "doigts de zinc" (Miller et al, 1985; Klug et Rhodes, 1987; Evans et Hollenberg, 1988). L'ensemble de la séquence est caractéristique de protéines nucléaires agissant comme facteurs de transcription. En raison de sa structure et de sa localisation, ce gène a été appelé $Z F Y$ (zinc finger $Y$-coded). 80 à $90 \%$ des mâles XX examinés possèdent ce segment d'ADN du chromosome $Y$, ainsi que les loci plus distaux. A l'inverse on ne connaît pas de mâles $X X$ positifs pour des sondes du chromosome $Y$ (mâles $X X Y(+))$ qui ne contiennent pas ZFY (Page et al, 1987c; Fergusson-Smith et Affara, 1988). Chez la souris, le chromosome $Y$ porte deux copies de ce gène (Zfy1 et Zfy2) (Mardon et al, 1989; Nagamine et al, 1989). Les deux gènes murins sont présents dans la région $S x r$, qui contient également $T d y$ (l'homologue murin de TDF) responsable de phénomènes d'inversion de sexe chez cet animal. II reste cependant à démontrer que $Z F Y(Z f y)$ et TDF (Tdy) ne sont qu'une seule et même entité. Alors que $Z F Y$ semble exprimé dans des tissus germinaux et somatiques, l'expression de Zfy1 et Zfy2 n'a été observée que dans le testicule adulte et non pas dans le testicule embryonnaire. De plus l'implication de $Z F Y$ 
reste à établir dans la majorité des cas de féminité $X Y$, et elle peut être pratiquement exclue de la masculinité et de l'hermaphrodisme $X X Y(-)$.

$Z F Y$ est fortement homologue à $Z F X$, un gène localisé sur le bras court du chromosome $X$ (Page et al, 1987c). II vient d'être montré que $Z F X$ échappe à l'inactivation qui affecte un des chromosomes $X$ dans les cellules femelles (SchneiderGädicke et al, 1989). Alors que son expression paraît relativement ubiquitaire, sa fonction demeure totalement inconnue. De façon inattendue, ZFX et ZFY correspondent à un gène autosomique chez les marsupiaux (Sinclair et al, 1988). Sans préjuger d'une éventuelle fonction, cette localisation est incompatible avec un rôle de déterminant testiculaire qui doit être exercée par un autre gène chez les marsupiaux. Ce serait parallèlement à une divergence récente dans l'évolution, consécutive à la translocation de ces gènes sur les chromosomes sexuels, que $Z F Y$ aurait acquis son hypothétique rôle de facteur de différenciation testiculaire.

L'évolution particulière des chromosomes sexuels a fait apparaître d'autres propriétés biologiques qui leur sont uniques et en rapport direct avec certaines anomalies de détermination du sexe.

\section{PROPRIÉTÉS DE LA RÉGION PSEUDOAUTOSOMIQUE}

Une étude quantitative du phénomène de recombination entre $X$ et $Y$ nous a montré que la région à proximité immédiate du télomère du bras court recombine avec TDF à une fréquence de $50 \%$, les autres loci recombinant plus rarement (Rouyer et al, $1986 \mathrm{a}, \mathrm{b})$. Aucune double recombinaison n'a été observée à ce jour (Page et al, 1987a; Weissenbach et al, 1987), alors que de tels doubles événements se produi- sent assez fréquemment dans la région pseudoautosomique de la souris (Soriano et al, 1987). L'absence de double recombinaison est par ailleurs confirmée par une parfaite additivité des taux de recombinaison inter-loci valide pour l'ensemble de la région (Rouyer et al, 1986a, b). Les résultats de cette étude quantitative montrent que la région pseudoautosomique est le siège d'un crossing-over unique et obligatoire se produisant en un point variant d'une méiose à l'autre. Une carte génétique peut être construite, soit à partir de la mesure directe des taux de recombinaison avec $T D F$, soit à partir des taux inter-loci. Les loci pseudoautosomiques s'ordonnent selon un gradient de liaison au sexe qui reflète directement la position d'un locus par rapport au bloc synténique constitué des régions spécifiques d'un chromosome sexuel. Plus un locus est proche du télomère du bras court, plus son taux de recombinaison avec TDF sera élevé et inversement.

Une comparaison des taux de recombinaison entre deux loci pseudoautosomiques fait ressortir des valeurs 10 à 20 fois plus élevées en méiose mâle (recombinaison $X Y$ ) qu'en méiose femelle (recombinaison $X / X)$ (Rouyer et al, 1986a, b). II fut proposé, il y a plus de cinquante ans, que la disjonction correcte des partenaires d'un bivalent méiotique pouvait être assurée chez les vertébrés par la formation d'un chiasma (Koller et Darlington, 1934). Dans le bivalent $X / Y$ le chiasma aurait à se former dans une zone particulièrement réduite - la région pseudoautosomique alors qu'il pourrait se produire en un point quelconque du bivalent $X / X$. Ceci pourrait aisément rendre compte d'une différence d'un ordre de grandeur entre les taux mâles et femelles dans cette région. A l'inverse, on pourrait supposer l'existence d'une inhibition de crossing-over entre deux chromosomes $X$ dans la région pseu- 
doautosomique. Une détermination précise des distances physiques nous a permis de trancher entre ces deux hypothèses.

L'analyse de grands fragments séparés par électrophorèse en champ pulsé nous a permis de dresser une carte physique de l'ensemble de la région pseudoautosomique. Celle-ci s'étend sur environ $2600 \mathrm{ki}$ lobases (Brown, 1988; Petit et al, 1988). En comparant cartes physique et génétique de la région pseudoautosomique, on observe qu'un $\mathrm{cM}$ en méiose mâle ( $1 \%$ de la recombinaison) correspond à une distance physique de $50 \mathrm{~kb}$ environ, alors qu'un $\mathrm{cM}$ correspond en moyenne à une distance de l'ordre de $1000 \mathrm{~kb}$ pour le reste du génome. Cette valeur est proche de celle que l'on trouve en méiose femelle dans la région pseudoautosomique. La forte différence des taux de recombinaison entre les deux sexes est donc la conséquence d'une très haute fréquence d'échanges génétiques dans cette région probablement due à sa petite taille.

\section{ÉCHANGES ANORMAUX ENTRE LES CHROMOSOMES X ET Y}

L'examen de cas d'inversion de sexe à l'aide de sondes pseudoautosomiques a également permis de vérifier l'origine chromosomique de la région pseudoautosomique paternelle, et par conséquent de tester la validité de l'hypothèse de FergusonSmith (1966), qui supposait un échange anormal entre les chromosomes sexuels. Comme $X$ et $Y$ subissent en apparence un crossing-over unique, on pouvait penser que ce crossing-over accidentel se produisait à la place de l'événement normal. On devait donc retrouver la totalité de la région pseudoautosomique du chromosome $Y$ sur un des chromosomes $X$ des mâles $\mathrm{XX}$ et l'absence complète des loci pseudo- autosomiques du chromosome $Y$ chez les femelles $X Y$. A l'inverse, la partie la plus distale du chromosome $X$ paternel devait être absente chez les mâles $X X$ et présente chez les femelles $X Y$ (fig 2).

II avait d'abord été montré que l'un des chromosomes $X$ était d'origine paternelle (Page et de la Chapelle, 1984). Une autre série d'observations mentionnée ci-dessus indiquait en outre que chez des mâles $X X$ le matériel provenant du chromosome $Y$ se retrouvait à l'extrémité du bras court d'un des deux chromosomes $X$ (Andersson et al, 1986; Buckle et al, 1987; Magenis et al, 1987). De plus des sondes spécifiques de la partie du chromosome $X$ immédiatement adjacente à la région pseudoautosomique nous ont permis de localiser les points de cassures de ces inversions de sexe sur le chromosome $X$.

\section{Masculinité $X X$}

Deux études portant au total sur une dizaine de mâles $X X Y(+)$ montrent que le réarrangement chromosomique résulte dans tous les cas analysés d'un échange anormal entre les parties terminales des bras courts des chromosomes $X$ et $Y$ paternels. Comme prévu, cet échange inégal se traduit par la perte d'une partie plus ou moins étendue de l'extrémité du bras court du chromosome $X$ associée à l'acquisition de la partie terminale du bras court du chromosome $Y$ (Page et al, 1987b; Petit et al, 1987). Dans quelques cas, la perte de l'extrémité de l'X est limitée à la partie distale de la région pseudoautosomique (fig 2C), mais le plus souvent elle s'étend dans la région $X$-spécifique (fig $2 B$ ). Les cas de masculinité $X X Y(-)$ analysés ne montrent pas d'échange anormal (Petit et al, 1987) et laissent supposer que l'inversion de sexe peut être indépendante de TDF. 


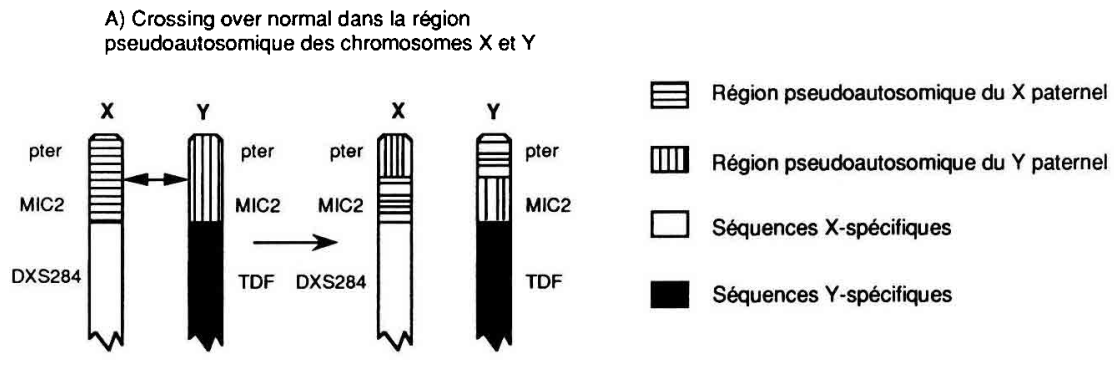

B) Crossing over anormal entre des régions spécifiques des chromosomes $X$ ef $Y$

C) Crossing over anormal entre la région pseudoautosomique du $X$ et des séquences $Y$-spécifiques
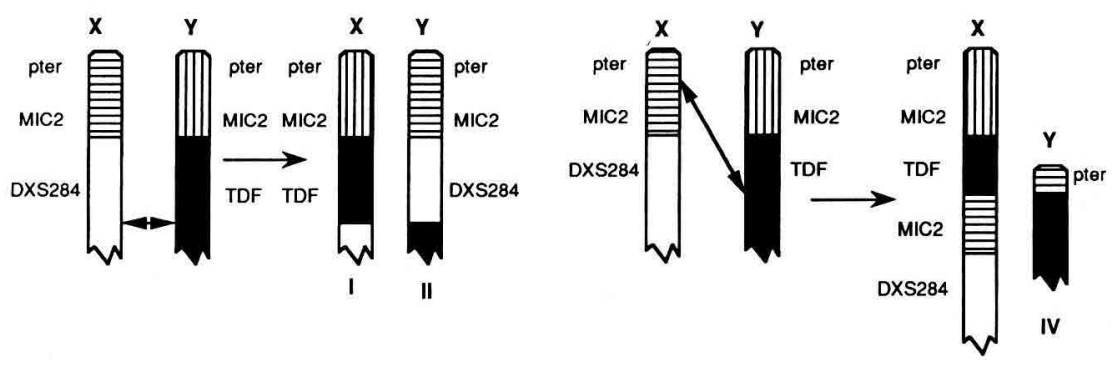

III

Fig 2. A) Crossing-over pseudoautosomique normal. B) et C) Crossing-over anormaux. Les chromosomes réarrangés produits par ces accidents méiotiques sont retrouvés chez les patients présentant des inversions de sexe. Chromosomes $X$ de types I (fréquent) et III (rare) présents chez les hommes $46, X X$. Chromosomes $Y$ de types II (rare) et IV (rare) chez les femmes $46, X Y$.

\section{Féminité $X Y$}

A côté d'une grande majorité de cas de femmes $X Y$ qui ne présentent pas de délétion du chromosome $Y$ décelable par les sondes moléculaires, on rencontre quelques patientes ayant perdu une partie plus ou moins importante du bras court. Ces dernières présentent en outre des stigmates du syndrome de Turner, en particulier un lymphœedème des extrémités (syndrome de Bonnevie-Ullich). Nous avons pu montrer par l'analyse familiale de deux cas, la perte de la région pseudoautosomique du chromosome $Y$ et l'acquisition, sur celui-ci, de la partie terminale du chromosome $X$ paternel (Levilliers et al, 1989). Les délétions du chromosome $Y$ de ces deux femmes résultent donc également d'un échange méiotique inégal entre les parties terminales des bras courts des chromosomes $X$ et $Y$ humains.

Conformément aux prédictions du modèle de l'échange terminal inégal, certains cas de féminité $X Y$ représentent donc la contrepartie exacte du type le plus répandu de mâles $X X Y(+)$ (fig 2B). On observe néanmoins une forte disparité entre la fréquence élevée $(1 / 20000)$ de mâles $X X$ $Y(+)$ et la faible incidence $(1 / 500000) d e$ 
femmes $X Y$ avec délétion du chromosome $Y$. Ces dernières étant affectées de stigmates du syndrome de Turner, celui-ci pourrait avoir une incidence sur la viabilité de ce type de femmes $X Y$. On sait en effet qu'une majorité des fœetus de caryotype $45, X$ (syndrome de Turner) est victime d'avortement spontané. Les cas analysés ci-dessus possèdent deux copies complètes de la région pseudoautosomique et permettent donc d'exclure de celle-ci la localisation de gène(s) "anti-Turner». A l'inverse, la partie délétée du chromosome $Y$ doit contenir un tel gène (Levilliers et al, 1989). II n'est pas exclu que la suppression du phénotype Turner chez les mâles normaux soit le résultat d'effets somatiques exercés par ZFY. Des résultats récents qui montrent que $Z F X$ échappe à l'inactivation sur le chromosome $X$ semblent même renforcer cette hypothèse. L'apparition d'un lymphœdème périphérique pourrait donc être due à l'absence de $Z F Y$ ou d'une copie de $Z F X$.

\section{Point de jonction $X-Y$ chez les mâles $X X Y(t)$}

L'analyse moléculaire d'une jonction entre les chromosomes $X$ et $Y$ chez un mâle $X X$ nous a montré que ces deux segments chromosomiques étaient reliés par une séquence d'ADN répétitif de type Alu (Rouyer et al, 1987). Le fait que l'on retrouve un élément Alu à cet emplacement sur chacun des chromosomes normaux montre que la fonction s'est produite entre ces deux éléments. L'analyse de séquence de la jonction suggère que celle-ci résulte d'une recombinaison homologue intervenue entre ces deux éléments Alu. Cette observation indique que l'échange anormal entre les deux chromosomes $X$ et $Y$ correspond selon toute vraisemblance à un accident du crossing-over normal, pro- voqué par une recombinaison homologue entre deux régions globalement hétérologues.

\section{ANTIGENE H-Y}

Bien que caractérisé depuis de nombreuses années, l'antigène $\mathrm{H}-\mathrm{Y}$ a fait l'objet de longues controverses et sa fonction demeure inconnue à ce jour. L'antigène $\mathrm{H}-\mathrm{Y}$ a d'abord été défini par une réaction de rejet de greffe comme antigène mineur d'histocompatibilité spécifique du sexe masculin et donc du chromosome $Y$ (Eichwald et Silmser, 1955). Par la suite H-Y a aussi été défini par une réaction sérologique et plus récemment par un essai faisant appel à des lymphocytes $T$ cytotoxiques. II est probable que l'antigène de transplantation soit identique à celui caractérisé par l'essai de prolifération des lymphocytes $T$ tueurs et distinct de l'antigène défini par sérologie. On a longtemps supposé que ce (ou ces) antigène(s) $\mathrm{H}-\mathrm{Y}$ étai(en)t étroitement associé(s) à la détermination du sexe. On avait en particulier corrélé la présence de l'antigène $H-Y$ avec le phénotype masculin des souris $X X S X r$, dont un chromosome $X$ porte un fragment submicroscopique du chromosome $Y$ comprenant les deux gènes Zfy. Au cours de croisements de souches porteuses du réarrangement chromosomique Sxr, on a constaté l'apparition de souris mutantes $X X S x r^{\prime}$ de phénotype masculin, ayant subi une délétion partielle de la région Sxr mais n'exprimant plus l'antigène $\mathrm{H}-\mathrm{Y}$ (McLaren et al, 1984). Ces résultats montrent donc clairement que l'antigène $\mathrm{H}-\mathrm{Y}$ n'est pas l'agent de différenciation testiculaire. II a pu être montré d'autre part que des souris $39, \mathrm{X}$ Sxr' ( $\mathrm{H}-\mathrm{Y}$ négatives) n'ont aucune spermatogénèse alors que des souris $39, X$ Sxr (H-Y positives) ont une spermatogénèse incomplète (Burgoyne et al, 1986). 
Cette observation suggère que $\mathrm{H}-\mathrm{Y}$ agirait au niveau de la spermatogénèse.

Des observations dissociant l'antigène $\mathrm{H}-\mathrm{Y}$, détecté par cytotoxicité des cellules $\mathrm{T}$, et la détermination du sexe ont aussi été faites chez l'homme. C'est ainsi qu'on a pu montrer que les mâles $X X Y(+)$ sont $H-Y(-)$ alors que les femmes $X Y$ avec délétion dans Yp sont $\mathrm{H}-\mathrm{Y}(+)$ (Simpson et al, 1987). Une cartographie plus précise de $\mathrm{H}-\mathrm{Y}$ place ce locus dans la partie proximale du bras long du chromosome $Y$, loin de TDF (fig 1). C'est aussi dans cette région qu'a été placé $A Z F$, un facteur contrôlant la fertilité chez l'homme (Tiepolo et Zuffardi, 1976).

\section{AUTRES HOMOLOGIES ENTRE X ET Y}

Au cours de l'analyse moléculaire du chromosome $Y$, plusieurs régions d'homologies entre les chromosomes $X$ et $Y$ ont été mises en évidence (fig 1). Ces régions permettent de reconstituer quelques-uns des événements majeurs qui ont déterminé l'évolution récente de ce chromosome. Les premières homologies observées remontent à l'isolement des premières sondes du chromosome $Y$. Des homologies de plus de $95 \%$ au niveau de la séquence nucléotidique ont été observées entre fragments d'ADN du milieu du bras long du chromosome $X(X q 13-q 24)$ et du bras court du chromosome $Y$ (Page et al, 1984; Geldwerth et al, 1985; Affara et al, 1986b). Ces séquences sont absentes du chromosome $Y$ des grands singes et des autres primates, le taux d'hétérogénéité entre les séquences $X$ et $Y$ permettant de dater le début de leur divergence entre 1 et 3 millions d'années (Page et al, 1984). Cette estimation confirme l'hypothèse d'une transposition d'un grand segment du bras long du chromosome $X$ sur le $Y$ chez un ancêtre de l'homme, après sa séparation des grands singes. Cette région d'homolo- gie est l'un des témoins les plus récents de réarrangements interchromosomiques qui ont jalonné l'évolution. Sur le chromosome $X$ cette région semble encore former un segment d'ADN continu. Mais cette continuité est interrompue au niveau du chromosome $Y$ où ce segment semble fragmenté en deux ou trois blocs de tailles inégales. Cette fragmentation n'est pas retrouvée sur tous les chromosomes $Y$ analysés et certains polymorphismes d'inversion mentionnés plus hauts pourraient en fait correspondre à un chromosome $Y$ ancestral, n'ayant pas encore subi de réarrangement dans ce bloc d'homologie.

D'autres séquences montrant une homogénéité supérieure à $99 \%$ ont été observées entre Xq28 et $\mathrm{Yq}$ (Cooke et al, 1984; Bickmore et Cooke, 1987; Arveiler et al, 1989). Ces séquences sont également absentes sur le $Y$ des pongidés et représentent une transposition plus récente. $A$ côté de ces homologies dues à des transpositions de $X$ vers $Y$ on retrouve des séquences beaucoup plus divergées $(80 \%$ d'homologie) présentes sur les deux chromosomes sexuels de nombreux primates. Ces séquences sont localisées en Xp22.3 d'une part et le plus souvent en Yq11 d'autre part (pour une revue, voir Davies et al, 1987). La localisation de l'homologie dans une région flanquant la région pseudoautosomique sur le chromosome $X$ suggère que ces séquences ont pu faire partie d'une région pseudoautosomique plus vaste, dont la partie proximale a été isolée sur le chromosome $Y$ à la suite d'une inversion péricentrique (Fraser et al, 1987; Yen et al, 1988). Ces séquences pourraient encore être pseudoautosomiques chez les lémuriens (Koenig et al, 1985; Yen et al, 1988). Plusieurs gènes ont été localisés dans la région Xp22.3, notamment ceux codant pour l'antigène érythrocytaire $\mathrm{Xg}$, la stéroïde sulfatase (STS) et l'amélogénine (AMG, protéine de l'émail dentaire). Les deux premiers échappent à 
l'inactivation, qui néanmoins affecte partiellement STS. On retrouve des séquences apparentées à STS (Fraser et al, 1987; Yen et al, 1988) et AMG (Lau et al, 1989) sur le bras long du chromosome $Y$. On peut donc penser que la non inactivation de ces gènes liés au $X$ représente un vestige de leur caractère pseudoautosomique. Dans cette hypothèse, STS serait un gène en voie de devenir inactivable sur le chromosome $X$.

\section{CONCLUSION}

Depuis la mise en évidence de séquences d'ADN du chromosome $Y$ chez certains hommes $X X$ en 1984, les techniques de biologie moléculaire ont fait faire un bond en avant à notre connaissance du chromosome $Y$ humain. La cartographie du chromosome a rapidement débouché sur la découverte d'un gène candidat pour la fonction de détermination primaire du sexe, et l'obtention de sondes pseudoautosomiques a permis de valider le modèle de l'échange génétique normal ou anormal entre les chromosomes $X$ et $Y$ à la méiose. Ainsi les bases structurales des phénomènes d'inversion de sexe, hommes $X X$ et femmes $X Y$, sont maintenant connues pour une grande partie des cas. Néanmoins les fonctions biochimiques du chromosome $Y$ restent largement inconnues puisque le clonage du gène $Z F Y$ n'a toujours pas permis d'affirmer son identité avec TDF, et a fortiori d'élucider la cascade de régulations génétiques responsables de la différenciation masculine de l'embryon.

\section{RÉFÉRENCES}

Affara NA, Ferguson-Smith MA, Tolmie J, Kwok K, Mitchell M, Jamieson D, Cooke A, Florentin $L$ (1986a) Variable transfer of $Y$-specific sequences in XX males. Nucleic Acids Res $14,5375-5387$
Affara NA, Florentin L, Morrison N, Kwok K, Mitchell $M$, Cooke $A$, Jamieson $D$, Glasgow $L$, Meredith L, Boyd E, Ferguson-Smith MA (1986b) Regional assignment of $Y$-linked DNA probes by deletion mapping and their homology with $X$ chromosome and autosomal sequences. Nucleic Acids Res 14, 53535373

Affara NA, Ferguson-Smith MA, Magenis RE, Tolmie JL, Boyd E, Cooke A, Jamieson D, Kwok K, Mitchell M, Snadden L (1987) Mapping the testis determinants by an analysis of $Y$-specific sequences in males with apparent $X X$ and $X O$ karyotypes and females with $X Y$ karyotypes. Nucleic Acids Res 15, 73257342

Andersson $M$, Page DC, de la Chapelle A (1986) Chromosome Y-specific DNA is transferred to the short arm of $X$ chromosome in human XX males. Science 233, 786-788

Arveiler B, Vincent A, Mandel JL (1989) Toward a physical map of the Xq28 region in man: linking color vision, G6PD, and coagulation factor VIII genes to an $X-Y$ homology region. Genomics 4, 460-471

Bickmore WA, Cooke HJ (1987) Evolution of homologous sequences on the human $X$ and $Y$ chromosomes, outside of the meiotic pairing segment. Nucleic Acids Res 15, 6261-6271

Brondum Nielsen $K$, Schwartz M, Sardemann H (1988) Investigation of three XX males by cytogenetic and DNA analyses. Hum Genet 78, 179-182

Brown WRA (1988) A physical map of the human pseudoautosomal region. EMBO J 7 , 2377-2385

Buckle VJ, Boyd Y, Fraser N, Goodfellow PN, Wolfe J, Craig IW (1987) Localization of $Y$ chromosome sequences in normal and $X X$ males. J Med Genet 24, 197-203

Burgoyne PS (1982) Genetic homology and crossing-over in the $X$ and $Y$ chromosomes of mammals. Hum Genet 61, 85-90

Burgoyne PS, Levy ER, McLaren A (1986) Spermatogenic failure in male mice lacking $\mathrm{H}-\mathrm{Y}$ antigen. Nature 320, 170-172

Cooke HJ, Brown AR, Rappold GA (1984) Closely related sequences on human $X$ and $Y$ chromosomes outside ther pairing region. Nature 311, 259-261

Cooke HJ, Brown WRA, Rappold GA (1985) Hypervariable telomeric sequences from the human sex chromosomes are pseudoautosomal. Nature 317, 687-692

Davies KE, Mandel JL, Weissenbach J, Fellous $M$ (1987) Report of the committee on the genetic constitution of the $X$ and $Y$ chromosomes. Cytogenet Cell Genet 46, 277-315 
de la Chapelle $A$ (1981) Etiology of maleness in XX men. Hum Genet 58, 105-116

Disteche CM, Casanova $M$, Saal $H$, Friedman C, Sybert V, Graham J, Thuline H, Page DC, Fellous $M$ (1986) Small deletions of the short arm of the $Y$ chromosome in 46, $X Y$ females. Proc Natl Acad Sci USA 83, 7841-7844

Eichwald EJ, Silmser CR (1955) Untitled communication. Transplant Bull 2, 148-149

Evans HJ, Buckton KE, Spowart G, Carothers $A D$ (1979) Heteromorphic $X$ chromosomes in 46, XX males: evidence for the involvement of $X-Y$ interchange. Hum Genet 49, 11-31

Evans RM, Hollenberg SM (1988) Zinc fingers: guilt by association. $\mathrm{Cel} / 52,1-3$

Ferguson-Smith MA (1966) $X-Y$ chromosomal interchange in the etiology of true hermaphroditism and of XX Klinefelter's syndrome. Lancet, ii, 475-476

Ferguson-Smith MA, Affara NA (1988) Accidental $X-Y$ recombination and the aetiology of $X X$ males and true hermaphrodites. Phil Trans $A$ Soc London B322, 133-144

Ford CE, Jones KW, Polani PE, de Almeida JC, Briggs JH (1959) A sex chromosome anomaly in a case of gonadal dysgenesis (Turner's syndrome). Lancet $i, 711-713$

Fraser N, Ballabio A, Zollo M, Persico G, Craig I (1987) Identification of incomplete coding sequences from steroid sulphatase on the human $Y$ chromosome: evidence for an ancestral pseudoautosomal gene? Development 101 suppl, 127-132

Friend SH, Bernards R, Rogelj S, Weinberg RA, Rapaport JM, Alberts DM, Dryja TP (1986) A human DNA segment with properties of the gene that predisposes to retinoblastoma and osteosarcoma. Nature 326, 643-646

Geidwerth D, Bishop C, Guellaën $G$, Koenig $M$, Vergnaud G, Mandel JL, Weissenbach J (1985) Extensive DNA sequence homologies between the human $Y$ and the long arm of the $X$ chromosome. EMBO J 4, 1739-1743

Goodfellow PJ, Darling SM, Thomas NS, Goodfellow PN (1986) A pseudoautosomal gene in man. Science 243, 740-743

Guellaën G, Casanova M, Bishop C, Geldwerth $D$, André $G$, Fellous $M$, Weissenbach $J$ (1984) Human $X X$ males with $Y$ single-copy DNA fragments. Nature 307, 172-173

Jacobs PA, Strong JA (1959) A case of human intersexuality having a possible $X X Y$ sex determining mechanism. Nature 183, 302-303

Klug A, Rhodes D (1987) 'Zinc fingers': a novel protein motif for nucleic acid recognition. Trends Biochem Sci 12, 464-469

Koller PC, Darlington CD (1934) The genetical and mechanical properties of the sex chro- mosomes. I. Rattus norvegicus, male. $J$ Genet 29, 159-173

Koenig M, Moisan JP, Heilig R, Mandel JL (1985) Homologies between $X$ and $Y$ chromosomes detected by DNA probes: localisation and evolution. Nucleic Acids Res 13, 5485-5501

Lau EC, Mohandas TK, Shapiro LJ, Slavkin HC, Snead ML (1989) Human and mouse amelogenin gene loci are the sex chromosomes. Genomics 4, 162-168

Levilliers J, Quack B, Weissenbach J, Petit C (1989) Exchange of terminal portions of $X$ and $Y$-chromosomal short arms in human $X Y$ females. Proc Natt Acad Sci USA 86, 29962300

Magenis RE, Webb MJ, McKean RS, Tomar D, Allen LJ, Kammer H, Van Dyke DL, Lovrien E (1982) Translocation $(X ; Y)$ (p22.33;p11.2) in XX males: etiology of male phenotype. Hum Genet 62, 271-276

Magenis RE, Casanova M, Fellous $M$, Olson S, Sheehy R, Tomar D (1987) Further cytologic evidence for $X p-Y p$ translocation in $X X$ males using in situ hybridization with $Y$ derived probe. Hum Genet 75, 228-233

Mardon G, Mosher R, Disteche CM, Nishioka Y, McLaren A, Page DC (1989) Duplication, deletion, and polymorphism in the sex determining region of the mouse $Y$ chromosome. Science 243, 78-80

Mardon G, Page DC (1989) The sexdetermining region of the mouse $Y$ chromosome encodes a protein with a highly acidic domain and 13 zinc fingers. Cell 56, 765-770

McLaren A, Simpson E, Tomonari K, Chandler $P$, Hogg $H$ (1984) Male sexual differentiation in mice lacking $\mathrm{H}-\mathrm{Y}$ antigen. Nature 312 , 552-555

Miller J, McLachlan AD, Klug A (1985) Repetitive zinc-binding domains in the protein transcription factor IIIA from Xenopus oocytes. EMBO J 41, 1609-1614

Monaco AP, Neve RL, Coletti-Feener C, Bertelson CJ, Kurnitt DM, Kunkel LM (1986) Isolation of candidate cDNAs for portions of the Duchenne muscular dystrophy gene. Nature 323, 646-650

Müller U, Donlon T, Schmid M, Fitch N, Richer CL, Lalande M, Latt SA (1986) Deletion mapping of the testis determining locus with DNA probes in $46, X X$ males and in $46, X Y$ and $46, X, d i c(Y)$ females. Nucleic Acids Res 14 , 6489-6515

Nagamine CM, Chan K, Kozak CA, Lau YF (1989) Chromosome mapping and expression of a putative testis-determining gene in mouse. Science 243, 80-83 
Orkin SH (1986) Reverse genetics and human disease. Cell 47, 845-850

Page DC, de la Chapelle A (1984) The parental origin of $X$ chromosomes in $X X$ males determined using restriction fragment length polymorphisms. Am J Hum Genet 36, 565-575

Page DC, Harper ME, Love J, Botstein D (1984) Occurrence of a transposition from the $X$ chromosome long arm to the $Y$ chromosome short arm during human evolution. Nature 311, 119-123

Page DC, Bieker K, Brown LG, Hinton S, Leppert $M$, Lalouel JM, Lathrop $M$, NystromLahti M, de la Chapelle A, White R (1987a) Linkage, physical mapping, and DNA sequence analysis of pseudoautosomal loci on the human $X$ and $Y$ chromosomes. Genomics 1, 243-256

Page DC, Brown LG, de la Chapelle A (1987b) Exchange of terminal portions of $X$ - and $Y$ chromosomal short arms in human $X X$ males. Nature 328, 437-440

Page DC, Mosher R, Simpson EM, Fisher EMC, Mardon G, Pollack J, McGillivray B, de la Chapelle A, Brown LG (1987c) The sexdetermining region of the human $Y$ chromosome encodes a finger protein. Cell 51, 1091-1104

Petit C, de la Chapelle A, Levilliers J, Castillo S, Nö̈l B, Weissenbach J (1987) An abnormal terminal $X-Y$ interchange accounts for most but not all cases of human $X X$ maleness. Cell 49, 595-602

Petit C, Levilliers J, Weissenbach J (1988) Physical mapping of the human pseudoautosomal region; comparison with genetic linkage map. EMBO J 7, 2369-2376

Rosenfeld RG, Luzzatti L, Hintz RL, Miller OJ, Koo GC, Wachtel SS (1979) Sexual and somatic determinants of the human $Y$ chromosome: studies in a $46, X Y \mathrm{P}^{-}$phenotypic female. Am J Hum Genet 31, 458-477

Rouyer F, Simmler MC, Johnsson C, Vergnaud G, Cooke HJ, Weissenbach J (1986a) A gradient of sex linkage in the pseudoautosomal region of the human sex chromosomes. $\mathrm{Na}$ ture 319, 291-295

Rouyer F, Simmler MC, Vergnaud G, Johnsson C, Levilliers J, Petit $\mathbf{C}$, Weissenbach $J$ (1986b) The pseudoautosomal region of the human sex chromosomes. Cold Spring Harbor Symp Quant Biol 51, 221-228

Rouyer F, Simmler MC, Page DC, Weissenbach $J$ (1987) A sex chromosome rearrangement in a human $X X$ male caused by Alu-Alu recombination. Cell 51, 417-425

Scherer G, Schempp W, Fraccaro M, Bausch E, Bigozzi V, Maraschio P, Montali E, Simoni G,
Wolf $U$ (1989) Analysis of two 47, XXX males reveals $X-Y$ interchange and maternal or paternal nondisjunction. Hum Genet 81, 247251

Schneider-Gädicke A, Beer-Romero P, Brown LG, Nussbaum R, Page DC (1989) ZFX has a gene structure similar to $Z F Y$, the putative human sex determinant, and escapes $X$ inactivation. Cell 57, 1247-1258

Simmler MC, Rouyer F, Vergnaud G, NyströmLahti M, Ngo KY, de la Chapelle A, Weissenbach J (1985) Pseudoautosomal DNA sequences in the pairing region of the human sex chromosomes. Nature 317, 692-697

Simpson E, Chandler P, Goulmy E, Disteche CM, Ferguson-Smith MA, Page DC (1987) Separation of the genetic loci for the H-Y antigen and for testis determination on human Y chromosome. Nature 326, 876-878

Simpson JL, Christakos AC, Horwith M, Silveraman FS (1971) Gonadal dysgenesis in individuals with apparently normal chromosomal complements: tabulation of cases and compilation of genetic data. Birth Defects 7, 215228

Sinclair AH, Foster JW, Spencer JA, Page DC, Palmer M, Goodfellow PN, Marshall Graves JA (1988) Sequences homologous to ZFY, a candidate human sex determining gene, are autosomal in marsupials. Nature 336, 780783

Soriano P, Keitges EA, Schorderet DF, Harbers K, Gartler SM, Jaenisch R (1987) High rate of recombination and double cross-overs in the mouse pseudoautosomal region during male meiosis. Proc Natl Acad Sci USA 84, 7218-7220

Tiepolo L, Zuffardi O (1976) Localization of the factors controlling spermatogenesis in the non-fluorescent portion of the human $Y$ chromosome long arm. Hum Genet 34, 119-124

Vergnaud G, Page DC, Simmler MC, Brown L, Rouyer F, Noël B, Botstein D, de la Chapelle A, Weissenbach $\mathrm{J}$ (1986) A deletion map of the human $Y$ chromosome based on DNA hybridization. Am J Hum Genet 38, 109-124

Weissenbach J, Levilliers J, Petit C, Rouyer F, Simmler MC (1987) Normal and abnormal interchanges between the human $X$ and $Y$ chromosomes. Development 101 suppl, 6774

Yen PH, Marsh B, Allen E, Tsaï SP, Ellison J, Connolly L, Neiswanger K, Shapiro LJ (1988) The human $X$-linked steroid sulfatase gene and a $Y$-encoded pseudogene: evidence for an inversion of the $Y$ chromosome during primate evolution. Cel/55, 1123-1135 